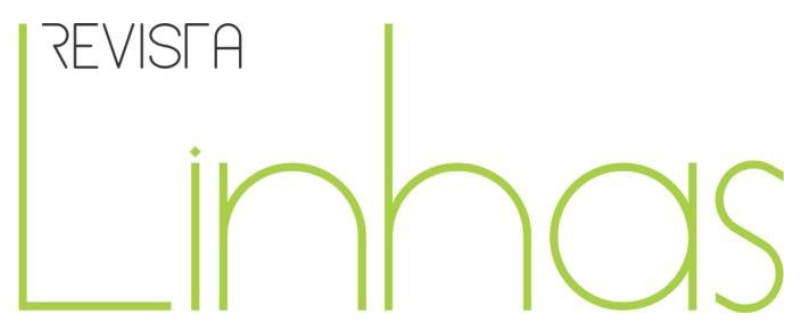

\title{
La Educación como un Derecho: Chile en tiempos de pandemia
}

\section{Resumen}

La pandemia derivada del nuevo Covid-19, ha marcado un antes y un después en distintas esferas de la vida de las personas y sus comunidades. El sistema educativo no ha sido la excepción y para reflexionar en torno a ello el presente artículo aborda la realidad educativa chilena en los niveles de primera infancia, primaria y secundaria. Se analiza el rol del Estado chileno para cautelar la educación para todos y todas en el marco internacional de los derechos humanos y luego se revisan las principales acciones del Ministerio de Educación de Chile para enfrentar la crisis. En el mismo sentido, desde la mirada de los y las docentes se aborda su accionar pedagógico desde la perspectiva de la entrega del servicio educativo a estudiantes de hogares con y sin conectividad y con y sin acceso a las tecnologías digitales. Finalmente se concluye con aprendizajes y desafíos para el Estado y el sistema educativo.

Palabras clave: Educación y pandemia. Derecho a la educación. Tecnologías y educación. Educación virtual. Educación a distancia.

\author{
Roberto Canales-Reyes \\ Universidad de Los Lagos - \\ Chile \\ rcanales@ulagos.cl
}

\footnotetext{
Para citar este artigo:

CANALES-REYES, Roberto. La Educación como un Derecho: Chile en tiempos de pandemia. Revista Linhas. Florianópolis, v. 22, n. 48, p. 106-121, jan./abr. 2021.
} 


\section{Education as a Right: Chile in times of pandemic}

\begin{abstract}
The pandemic derived from the Covid-19, has marked a before and after in different spheres of the lives of people and their communities. The educational system has not been the exception and to reflect on this, this article addresses the Chilean educational reality at the early childhood, primary and secondary levels. The role of the Chilean State to protect education for all in the international framework of human rights is analyzed and then the main actions of the Chilean Ministry of Education to face the crisis are reviewed. In the same sense, from the perspective of the teachers, their pedagogical actions are approached from the perspective of the delivery of the educational service to students from homes with and without connectivity and with and without access to digital technologies. Finally, it concludes with lessons learned and challenges for the State and the educational system.
\end{abstract}

Keywords: Education and pandemic. Right to education. Technologies and education. Virtual education. Distance education.

\section{Educação como um Direito: Chile em tempos de pandemia}

\begin{abstract}
Resumo
A pandemia derivada do coronavírus SARS-Cov2, a Covid-19, marcou um antes e um depois em diferentes esferas da vida das pessoas e de suas comunidades. O sistema educacional não tem sido exceção e, para refletir sobre isso, este artigo aborda a realidade educacional chilena nos níveis da primeira infância, fundamental e médio. Analisa-se o papel do Estado chileno na proteção da educação para todos no âmbito internacional dos direitos humanos e, a seguir, são revistas as principais ações do Ministério da Educação do Chile para enfrentar a crise. No mesmo sentido, na perspectiva dos professores, suas ações pedagógicas são abordadas na perspectiva da prestação do serviço educativo a alunos de residências com e sem conectividade e com e sem acesso às tecnologias digitais. Por fim, conclui com lições aprendidas e desafios para o Estado e o sistema educacional.
\end{abstract}

Palavras-chave: Educação e pandemia. Direito à educação. Tecnologias e educação. Educação virtual. Educação a distância. 


\section{El Estado chileno y el derecho a la educación}

La Declaración Universal de los Derechos Humanos, en su artículo 26, numerales 1, 2 y 3, consagra la Educación como un Derecho, indicando que su cobertura debe ser universal y gratuita, al menos en lo concerniente a la instrucción básica elemental y media o fundamental, indicando que "la educación tendrá por objeto el pleno desarrollo de la personalidad humana y el fortalecimiento del respeto a los derechos humanos y a las libertades fundamentales" (DUDH, 1948).

El Estado es el primer titular de obligaciones en materias de derecho internacional, luego las leyes y las políticas nacionales deben detallar cómo se cumplirán dichas obligaciones a nivel nacional, provincial y local.

En el transcurso del año 2020 en contexto de pandemia, se suspendieron las clases presenciales de todos los establecimientos educacionales de Chile, afectando a 3,7 millones de estudiantes distribuidos en 13.723 establecimientos de educación Parvularia, básica y Media (MINEDUC, 2021).

El Estado chileno, a pesar de los esfuerzos centralizados que se hicieron, puso en cuestión el derecho de todos y todas a la Educación, quedando al debe la entrega de las condiciones mínimas para su funcionamiento en todo el territorio nacional, contrario a las características que señala el Pacto Internacional de Derechos Económicos, Sociales y Culturales (DESC, 1966, ratificado por Chile en 1972) relacionado a la disponibilidad, accesibilidad, aceptabilidad y adaptabilidad de los derechos, entre ellos el de la educación.

En términos de la disponibilidad, que implica el que debe haber instituciones y programas en cantidad suficiente para acceder a la prestación y atender a los factores que condicionan su implementación, el Estado chileno debió garantizar calidad y equidad en el servicio educativo, no obstante, la situación fue discutible. Antes de la pandemia, en tiempos de normalidad y presencialidad, de alguna forma se aspiraba a entregar condiciones formativas, infraestructurales y sociales similares a todos los establecimientos educacionales públicos del país y a los estudiantes que accedían a ellos; sin embargo, con la pandemia, el distanciamiento social y el reemplazo de lo presencial por la educación virtual, no existieron los programas, ni las condiciones mínimas para acceder en igualdad 
de condiciones a la educación. Esto repercutió ampliando las brechas de desigualdad y disponibilidad, sobre todo en las regiones y los sectores rurales apartados de la metrópoli, territorios con escasa conexión a internet y acceso a las tecnologías digitales.

Un estudio realizado por la Fundación 99 junto a la Coordinación Nacional de Educación Rural del Ministerio de Educación, Oficina de Estudios y Políticas Agrarias del Ministerio de Agricultura y la Agrupación de Profesores y Profesoras Rurales de Chile, mostró cómo vivieron el año escolar 2020 cerca de 1.730 directivos, docentes y asistentes de la educación, evidenciando que un $34 \%$ de los profesores rurales nunca pudo hacer clases (presenciales o virtuales) y, en el caso de las escuelas multigrado -donde en una misma clase se les enseña a niños de distintos niveles-, la cifra sube a un $42 \%$.

Por otro lado, se suma a esto, las condiciones desventajosas en la cuales debieron desempeñarse profesores y profesoras en el territorio nacional, quienes en su gran mayoría financiaron a nivel personal, de bolsillo propio, planes de datos para acceder a internet, infraestructura y recursos tecnológicos para realizar docencia, entre ellos teléfonos celulares, computadores personales, tablet, etc. Así, “con el cierre de los establecimientos educacionales, esas condiciones mínimas comunes desaparecen por las desiguales condiciones de educabilidad que presentan los hogares y los territorios, particularmente las brechas digitales, de conectividad, acceso efectivo a internet y a los diversos grados de alfabetización que tiene la población para hacer uso de las herramientas digitales, impactando diferencialmente en el ejercicio del derecho a la educación durante la pandemia" (INSTITUTO NACIONAL DE DERECHOS HUMANOS DE CHILE [INDH], 2020).

Escuelas, liceos y universidades cerraron sus dependencias durante todo el año 2020, producto de la pandemia y en ese sentido, la segunda característica de los DESC anunciada, accesibilidad a la educación, la cual busca asegurar condiciones de accesibilidad material y/o económica a los servicios, implica poner en práctica el principio de igualdad y no discriminación, lo cual se encuentra seriamente cuestionado, toda vez que al implementar la educación a distancia, las familias de escasos recursos han visto mermada la posibilidad que sus hijos e hijas accedan regularmente a las clases en línea, recibir las guías de aprendizaje, recursos digitales, materiales educativos y/o cápsulas educativas virtuales. 
El Estado no ha asegurado las condiciones materiales ni económicas para que todos y todas accedan de igual manera a la educación, quedando en tela de juicio además, el principio de igualdad y no discriminación, toda vez que los niveles de desigualdad se han profundizado en estos nuevos escenarios, existiendo un número importante de estudiantes sin posibilidades de participar de los procesos formativos, producto de la escasa conexión a la red de datos y sin recursos económicos para disponer de la tecnología necesaria para participar de las sesiones de clases sincrónicas.

En momentos de crisis, de aislamiento social y de confinamiento, pasar de la presencialidad en educación a la virtualidad, ha significado un nuevo reto y desafío; no es sólo desde el punto de vista del proceso de enseñanza y aprendizaje, sino también de las brechas a nivel de oportunidades de accesibilidad y tecnológicas que se presentan entre los y las estudiantes para conectarse a la dinámica del proceso educativo (CANALES; SILVA, 2020).

En cuanto a la aceptabilidad, que dice relación con que los servicios deben ser pertinentes y de buena calidad en función de las características, necesidades y expectativas del grupo al que se dirige, se puede indicar que los servicios entregados por el Estado chileno y establecimientos privados en el ámbito de la educación, no han sido pertinentes ni de buena calidad. Esto se verifica especialmente en el ámbito público, donde ha habido escasa formación continua contextualizada para los y las docentes, especialmente en estrategias metodológicas y modalidades a distancia, con poca disponibilidad de materiales y recursos pedagógicos digitales según especialidad, debiendo enfrentar la situación con sistemas de autoformación e innovación en función del esfuerzo y compromiso personal y colectivo de los profesores y profesoras.

En relación al principio de adaptabilidad, que implica que las prestaciones deberán adecuarse a las necesidades territoriales, culturales y socio-históricas de sociedades y comunidades, los servicios educativos entregados por el Estado de Chile tampoco han estado a la altura de las necesidades territoriales, adoleciendo de condiciones mínimas de acceso a este derecho, presentando alternativas desiguales a los distintos estudiantes en función del territorio en que habitan, tanto a nivel de metrópolis como en los territorios más alejados y remotos, o bien por la situación precaria económica familiar. El ejemplo más 
frecuente está dado por familias que debieron compartir el único celular o computador con conexión a internet existente en el hogar, organizándose para su utilización a través de turnos durante la jornada. Cabe recordar que los adultos en forma paralela realizan teletrabajo y por su lado los estudiantes deben conectarse a las clases y actividades escolares sincrónicas.

De esta forma, en el ámbito del derecho a la educación, se pueden observar serias dudas en relación a que el Estado chileno haya destinado el máximo de recursos disponibles para enfrentar la crisis y el acceso a la educación para todas y todos, la pandemia del coronavirus ha contribuido a agudizar las diferencias contextuales, sociales, culturales y políticas que abarcan desde problemas de conectividad a la calidad de las prestaciones tecnológicas de los discentes (TEJEDOR et al. 2020).

En este contexto tan desfavorable para la educación, el sistema internacional de protección de Derechos Humanos ha llamado a los Estados a tomar todas las medidas que estén a su alcance y que se haga el máximo esfuerzo de recursos disponibles para asegurar un acceso asequible y equitativo a los servicios de Internet para todos y todas con fines educativos y a disponer de equipos técnicos básicos para los y las estudiantes, particularmente para aquellos que habitan en comunidades y regiones más pobres, situación que no ha hecho eco en Chile (INDH, 2020).

Así, el Estado chileno tuvo respuestas diferentes frente a los impactos de la pandemia en los distintos niveles educacionales que componen el sistema, ya sea por la oportunidad en que se tomaron las medidas, el uso de los recursos disponibles y la toma de medidas especiales para atender a grupos en situación de vulnerabilidad, para mitigar los efectos que la suspensión de clases presenciales tendría sobre el acceso a los procesos de aprendizaje y la permanencia en el sistema educativo (INDH, 2020). 


\section{Principales acciones de Ministerio de Educación chileno en tiempos de} pandemia

El Ministerio de Educación desplegó a nivel central, regional y provincial distintas estrategias, programas y acciones para apoyar la educación a distancia y adecuar la docencia y los aprendizajes al nuevo contexto. Entre las medidas se destacan las siguientes:

- Plataforma Aprendo en Línea, espacio educativo abierto a todas las dimensiones del aprendizaje https://www.curriculumnacional.cl/estudiantes/Ingreso

- Trabajo intensivo con textos escolares desde el hogar. Los estudiantes tuvieron acceso a todos los textos escolares, guías didácticas y cuadernos de actividades de todas las asignaturas y niveles, de manera online.

- Envío de material pedagógico físico en las asignaturas de Lenguaje y Matemática a estudiantes de Educación Parvularia hasta $4^{\circ}$ medio. Adicionalmente, se incorporó un cuadernillo para los apoderados y familias, donde se entregaron las soluciones de cada ejercicio y recomendaciones para favorecer el aprendizaje en el hogar.

- TVEduca en Chile. Canales de televisión abierta agrupados en Anatel y Arcatel, en conjunto con el Consejo Nacional de Televisión y el Ministerio de Educación, llegaron a un acuerdo para implementar una señal educativa en el país. https://www.tvn.cl/envivo/tveducachile/

- Biblioteca digital escolar. La Biblioteca Digital Escolar fue implementada en noviembre de 2018 y cuenta con más de 12.000 libros, incluyendo novelas y material educativo. https://bdescolar.mineduc.cl/

- Becas TIC entregando en 2020 más de 125.000 computadores con conexión a internet por once meses.

- Plan de lectoescritura digital. Iniciativa de fomento lector que permitió la creación de clubs de lectura de manera remota con libros de la Biblioteca Digital Escolar. https://planlectordigital.mineduc.cl/

- Aprendo FM. Cápsulas radiales alineadas al currículum, difundidas a través de 110 radios regionales a lo largo de Chile. Los contenidos abordaron lenguaje, ciencias, matemática, historia y bienestar (educación física y orientación) https://www.mineduc.cl/horariosaprendo-fm/ 
- Cuadernillos de juegos y experiencias de aprendizaje. Para atender a aquellos contextos en que el acceso de internet se consolida como una barrera, se desarrolló un cuadernillo de juegos y experiencias de aprendizaje basados en los Objetivos de aprendizaje priorizados para el tramo de Transición de las Bases Curriculares de Educación Parvularia.

- Apoyo para docentes. Alianza con Fundación Chile, Google y Microsoft para apoyar a los establecimientos en disponer, de forma gratuita, de los softwares GSuite para Educación y Office 365 A1

- Otros apoyos para las comunidades educativas en áreas fundamentales: alimentación, flexibilidad y bienestar socioemocional.

\section{La educación en tiempos de Pandemia. Una mirada desde los docentes}

El diagnóstico es similar en los distintos niveles educativos, especialmente en la enseñanza primaria y secundaria, donde las y los docentes enfrentaron con creatividad los desafíos planteados por el contexto, en el sentido de buscar formas y mecanismos para conectarse con sus pares y luego con estudiantes, padres y apoderados. Hubo que desplegar estrategias de distinto tipo, considerando las realidades heterogéneas existentes en el país, para ir adaptando en función de las condiciones económicas y sociales, las respuestas a las necesidades de la comunidad educativa con el propósito de realizar las actividades pedagógicas respectivas.

En primer término, se privilegió la interconexión social y el contacto humano con la comunidad educativa, evidenciando una alta preocupación por la salud general y mental de colegas y estudiantes, para luego, en materia educativa priorizar el trabajo con contenidos esenciales, planteando que "menos es más en tiempos de crisis" en materia de cobertura curricular.

La autoformación, el autoaprendizaje, la búsqueda de soluciones tecnológicas simples pero efectivas, fue la primera reacción de miles de docentes quienes trabajando en forma coordinada desplegaron estrategias pedagógicas conjuntas e interdisciplinarias entre asignaturas y con apoyo, en varios casos, de duplas psicosociales y Programas de 
Integración Escolar (PIE) para atender también a niños, niñas y adolescentes con necesidades educativas especiales.

El tránsito de lo presencial a lo virtual en el ámbito de la educación, no tuvo etapas intermedias, hubo que adaptarse rápidamente a las necesidades del contexto, lo que significó retos y desafíos para el sistema educativo, evidenciando y agudizando las brechas de desigualdad social.

A través de un diálogo con más de 50 directivos de Establecimientos Educacionales, docentes de la RED Ulagos (mayo, 2020) y con 55 docentes de educación para jóvenes y adultos (EPJA) de la décima región de Los Lagos en Chile (septiembre, 2020), se releva el importante rol que ha tenido el profesor y profesora demostrando un fuerte sello social y afectivo para atender a su comunidad educativa considerando los desafíos planteados por la pandemia. El sistema puso a prueba el papel de las familias en los procesos educativos de sus hijos e hijas, especialmente la mediación que hubo que realizar con los niños y niñas más pequeños, instancia en las cuales padres, madres y cuidadores cumplen la acción de articuladores con la escuela y docentes, facilitando el proceso de enseñanza y aprendizaje en casa, por cierto no ausente de dificultades.

En los diálogos ya referenciados, destaca la actitud de flexibilización por parte de los y las docentes frente a la enseñanza y el aprendizaje de sus estudiantes, en el entendido que la multiplicidad de casos y situaciones contextuales ameritan la comprensión y adaptación de los procesos formativos. En el mismo sentido, se opina en forma positiva la coincidencia en priorizar objetivos de aprendizajes y adecuación curricular estimulada por el Ministerio de Educación chileno, focalizando el trabajo en aprendizajes esenciales, objetivos integradores, significativos y de socialización.

Los profesores y profesoras perciben de forma favorable los espacios de reflexión, escucha activa y socialización con las y los estudiantes en torno a lo que se está vivenciando, quedando pendiente, según sus opiniones, el abordaje a nivel de orientaciones ministeriales para enfrentar el área de formación técnica profesional y por cierto, la educación de estudiantes jóvenes y adultos (EPJA), que están privados de libertad y en contextos de encierro. 
Dentro de las temáticas complejas expresadas por los profesores y profesoras se encuentra la dificultad de enfrentar la concentración, actitud, disposición y motivación de los estudiantes por aprender, considerando los riesgos de su contexto y núcleo familiar a nivel de salud, estabilidad laboral de los miembros de la familia y eventuales pérdidas de empleo por los prolongados tiempos de cuarentena.

A lo anterior se suma la dificultad que presentan las y los propios docentes para realizar retroalimentación de los aprendizajes, derivado de la imposibilidad de comunicación con todos y todas. Para ello resulta clave el acompañamiento y seguimiento continuo del proceso de aprendizaje personal y por equipos de trabajo, considerando que la retroalimentación oportuna evita la deserción por el estudio, mejora la motivación y actitud frente al aprendizaje, dejando en evidencia la eventual sobrecarga cognitiva depositada en el estudiante, debiendo desplegar estrategias de coordinación entre docentes que trabajan en los distintos cursos y niveles en el semestre lectivo para planificar en conjunto y en forma articulada los procesos evaluativos (CANALES; SILVA, 2020).

\section{Abordaje de la crisis en educación para hogares sin conectividad}

La reproducción de guías de aprendizaje en formato papel y su distribución cada quince días o una vez al mes, ha sido una de las actividades más desplegadas por los docentes en el país. Distintas han sido las estrategias para su distribución; en algunos sectores se han entregado junto a cajas de mercadería (raciones de desayuno y almuerzo equivalente a 15 días hábiles, y dirigidas a estudiantes beneficiarios de los programas de Alimentación Escolar y de Párvulos) proporcionadas por la Junta Nacional de Auxilio Escolar y Becas (JUNAEB del Mineduc), por otro lado padres, madres, apoderados y cuidadores acuden a los establecimientos educacionales a retirar el material educativo para que puedan trabajar sus hijos e hijas en casa.

En el ámbito de la educación de la primera infancia o educación parvularia se implementó, entre otras estrategias la "bolsa viajera", la cual es entregada cada quince días a las familias, en cuyo contenido se depositan las actividades a realizar por los niños y niñas, bolsa que luego retorna con las tareas resueltas. 
El estudio Fundación 99 (2020), respecto al tipo de estrategia de enseñanza utilizada, plantea que el 95\% de los directivos encuestados apuntó al envío de guías y tareas, y el $62 \%$ de los profesores optó por usar sus propios recursos, versus un $37 \%$ que utilizó el otorgado por el Mineduc.

Así también se hizo entrega de textos escolares de estudios, libros, novelas y literatura pertenecientes a los Centros de Recursos de Aprendizaje (CRA) de los establecimientos escolares, los cuales se renuevan en función de las lecturas derivadas de las tareas encomendadas.

Adicional a lo anterior, la comunicación telefónica de los profesores y profesoras jefe ha sido clave para relacionarse con los padres y apoderados en el caso de los estudiantes más pequeños y en forma directa con los estudiantes de cursos superiores, y fue una estrategia masiva y simple de implementar para concertar clases, reuniones de trabajo, resolver inquietudes de las guías de aprendizaje, entre otros aspectos.

Hubo despliegues también en terreno, visitas domiciliarias de docentes para acercar las guías de aprendizaje a los hogares de los estudiantes que no se logran conectar y así entregarles presencialmente el material educativo, como presentaciones o guías de trabajo en formato papel, toda vez que sin conectividad resultaba complejo el intercambio de correos electrónicos.

El estudio de la fundación 99, plantea que el 94\% de los profesores creen que en el 2020 los alumnos aprendieron menos que en un año normal, pero declararon que los estudiantes pudieron desarrollar habilidades como la autonomía (63\%), aprender de tecnología (53\%) y la creatividad (37\%) (FUNDACIÓN 99, 2020).

Es importante señalar que para el gremio docente, al enfrentar un año de desafíos y responsabilidades éticas y profesionales para darle continuidad a la educación a los grupos familiares ubicados en los distintos rincones del país y el estar conectado prácticamente gran parte del día desarrollando el teletrabajo, planificando, preparando materiales, adaptando las clases, realizando cápsulas educativas y revisando trabajos en línea, más las tareas propias del hogar, al término del semestre lectivo todo este exceso de trabajo confluyó en agobio, cansancio y estrés laboral. 


\section{Abordaje de la crisis para hogares con conectividad y tecnologías digitales}

En aquellos establecimientos educacionales cuyos estudiantes disponían de los recursos tecnológicos básicos para conectarse, la estrategia de enseñanza más utilizada fue la creación de cápsulas educativas con contenido digital, las cuales a través de pequeños videos grabados con los teléfonos celulares y luego subidos a la red internet, ya sea a un canal de YouTube o enviados por la red social WhatsApp, son derivados diariamente a los estudiantes. Estos videos generalmente son editados con distintas plataformas gratuitas y de pago tales como Loom, Movie Maker, Powtoon, Tik Tok, entre otras.

En el ámbito de la educación infantil, el Ministerio de Educación creó una app denominada “Mi jardín Junji” https://mijardinjunji.cl/, plataforma que permite conectar a las familias y a los niños y niñas con sus educadoras y asistentes de la educación.

Por otro lado, se masificó en forma exponencial la creación de grupos de trabajo en WhatsApp, ya sea entre colegas, apoderados y con, y entre los propios estudiantes, lo que permitía mensajería instantánea, intercambio de materiales educativos y la realización de videollamadas personales y en pequeños grupos para coordinarse, resolver dudas e inquietudes educativas.

Debido, en una primera instancia, a la ausencia de plataformas virtuales de aprendizaje digital, hubo docentes que crearon grupos de trabajo en Facebook, espacio en el cual fueron agregando a sus estudiantes para tener un contacto más fluido y lograr compartir contenidos digitales interactivos, documentos de trabajo y presentaciones varias.

En general, la estrategia más usada es la creación, por parte de los y las docentes, de guías de aprendizaje y cuadernillos pedagógicos que consisten en entrega de contenidos, actividades y ejercicios en formato digital que reciben los y las estudiantes a través de correo electrónico, redes sociales o plataformas virtuales de aprendizaje. $Y$, por cierto, siempre el contacto con los más pequeños se hizo a través de sus cuidadores, padres, madres y apoderados. 
Distintos establecimientos educacionales del país, en el segundo semestre del 2020, lograron instalar aulas virtuales de aprendizaje tipo Learning Management System (LMS), tanto gratuitas como de pago, tales como Web Class, Lirmi, Classroom, Moodle, y para realizar reuniones y clases virtuales con grupos pequeños se utilizó preferentemente las aplicaciones de Zoom, Hangouts-Meet, Jitsi-Meet, etc.

\section{A modo de conclusión: Aprendizajes y desafíos para el sistema educativo}

En función de lo vivido en el 2020, no ha sido fácil para los profesores y profesoras adaptarse a estos nuevos requerimientos y formatos virtuales, en el entendido que el docente se formó y aprendió de maestros y maestras en la clase presencial, tiene escasa o nula experiencia en estrategias de enseñanza y aprendizaje en contextos de virtualidad. (GARRISON; ANDERSON, 2005)

La enseñanza virtual requiere de un modelo pedagógico distinto al de la enseñanza presencial, que atienda y potencie la autonomía e independencia en el proceso de aprendizaje de los estudiantes. En este contexto, se han promovido nuevas formas de aprender, incorporando el aprendizaje ubicuo, el cual trasciende el contexto temporoespacial del espacio educativo tradicional y el aprendizaje flexible y abierto asociado a la introducción de los medios sociales en la educación (MARÍN, NEGRÉ, PÉREZ-GARCÍA, 2014; SALINAS, 2013).

En la actualidad, realizar docencia no sólo implica dominio de conocimientos específicos, capacidades y actitudes pedagógicas y didácticas, sino que además se hace relevante avanzar en el desarrollo profesional docente y la formación continua a nivel de Competencias Digitales Docentes, asumiendo modelos pedagógicos que soporten la educación virtual, en el entendido que son escasas las acciones formativas en tiempos de pandemia relacionadas a un modelo de formación online, el cual estructure y secuencie los contenidos y las actividades de aprendizaje, implementando metodologías activas y relevando al docente como mediador, fomentando la tutoría y la retroalimentación de los aprendizajes (CANALES; SILVA, 2020; SILVA, 2011). 
Desde la perspectiva de los y las estudiantes, las exigencias de estas nuevas modalidades han puesto a prueba sus competencias y habilidades digitales para enfrentar las exigencias del siglo XXI; se han visto fortalecidos en aprender y usar tecnologías, en desplegar mayor autonomía y creatividad, quedando pendiente trabajar la responsabilidad, autorregulación y autoaprendizaje.

Los principales desafíos que tienen el Estado y el sistema educativo son los siguientes:

- Dedicar el máximo de los recursos disponibles para asegurar a todas y todos el derecho a la educación, su promoción, respeto, protección y garantía.

- Desplegar acciones formativas para el desarrollo profesional docente en el ámbito pedagógico, de formación online y metodologías activas.

- Relevar la tutoría, retroalimentación en línea y la evaluación de los aprendizajes.

- Innovar en la docencia con apoyo de recursos digitales de aprendizaje y entornos virtuales de aprendizaje.

- Fomentar las competencias digitales docentes en los profesores y profesoras, y las habilidades del siglo XXI en los y las estudiantes.

- Estimular la creación de cursos virtuales con contenidos interactivos, cápsulas educativas y material de apoyo pertinente que permita el intercambio y la colaboración entre pares.

- Diagnosticar la competencia digital de los y las estudiantes es clave para desarrollar acciones formativas para la vida.

- A nivel de Establecimientos Educacionales:

- Definir un marco para abordar las competencias digitales docentes

- Implementar planes de desarrollo profesional docente y formación continua

- Implementar la priorización curricular en contexto de Covid-19

- Apoyar socioemocionalmente a los profesores y profesoras y sus estudiantes

- Promover una cultura digital a nivel de competencias ciudadanas, que fomente el respeto, la convivencia escolar, la ética y el autocuidado en ambientes digitales. 


\section{Referências}

CANALES, Roberto; SILVA, Juan. De lo presencial a lo virtual, un modelo para el uso de la formación en línea en tiempos de Covid-19. Educar em Revista, Curitiba, v. 36, 2020. Disponible en: DOI: http://dx.doi.org/10.1590/0104-4060.76140. Acceso en: 22 enero 2021.

FUNDACIÓN 99. Caracterización de la Educación Rural en Chile en contexto de pandemia por COVID-19. Santiago, 2020.

Disponible en: https://fundacion99.org/fundacion-99-lanza-su-primera-primera-encuestanacional-educacion-rural-en-chile-educacionrural2020-2/

Acceso en: 20 enero 2021.

GARRISON, D. Randy; ANDERSON, Terry. El e-learning en el siglo XXI: investigación y práctica. Barcelona: Octaedro, 2005.

INSTITUTO NACIONAL DE DERECHOS HUMANOS DE CHILE. Covid y derechos humanos en Chile: informe anual. Santiago, 2020.

MARÍN, Victoria; NEGRE, Francisca; PÉREZ-GARCÍAS, Adolfina. Entornos y redes personales de aprendizaje (plepln) para el aprendizaje colaborativo [Construction of the Foundations of the ple and pln for Collaborative learning]. Comunicar, [S.I.], v. 21, n. 42, p. 35-43, 2014. Disponible en: https://doi.org/10.3916/C42-2014-03. Acceso en: 20 enero 2021.

MINEDUC. Educación en Pandemia. Principales medidas del Ministerio de Educación de Chile en 2020. Santiago: Ministerio de Educación de Chile, 2021.

Disponible en: https://www.mineduc.cl/wp-

content/uploads/sites/19/2021/01/BalanceMineduc2020.pdf. Acceso en: 28 enero 2021.

ONU. Asamblea General. Pacto Internacional de Derechos Económicos, Sociales y Culturales. Adoptado y abierto a la firma, ratificación y adhesión por la Asamblea General en su resolución 2200 A (XXI). [S.I.]: Naciones Unidas, 1966. (Serie de Tratados). v. 993, p. 3. Disponible en: https://www.refworld.org.es/docid/4cof50bc2.html. Acceso en: 28 enero 2021

SALINAS, Javier. Enseñanza flexible y aprendizaje abierto, fundamentos clave de los ples. En: CASTAÑEDA, Linda; ADELL, Jordi (ed.). Entornos personales de aprendizaje: claves para el ecosistema educativo en Red. Alcoy: Marfil, 2013. p. 53-70.

SILVA, Juan. Diseño y moderación de entornos virtuales de aprendizaje. Barcelona: Editorial UOC, 2011.

TEJEDOR, Santiago; CERVI, Laura; TUSA, Fernanda; PAROLA, Alberto. Educación en tiempos de pandemia: reflexiones de alumnos y profesores sobre la enseñanza virtual universitaria en España, Italia y Ecuador. Revista Latina de Comunicación Social, [S.I.], v. 
78, p. 1-21, 2020. Disponible en: https://www.doi.org/10.4185/RLCS2020-1466. Acceso en: 28 enero 2021.

Recebido em: 08/02/2021 Aprovado em: 28/02/2021

Universidade do Estado de Santa Catarina - UDESC Programa de Pós-Graduação em Educação - PPGE

Volume 22 - Número 48 - Ano 2021 revistalinhas@gmail.com 